\title{
EXTRACTION KICKERS AND MODULATORS FOR THE ADVANCED HYDROTEST FACILITY*
}

\author{
P. L. Walstrom, Los Alamos National Laboratory, Los Alamos, NM 87545, USA \\ E. G. Cook, Lawrence Livermore National Laboratory, Livermore, CA, 94550, USA
}

\begin{abstract}
In order to exploit the full potential of the Advanced Hydrotest Facility (AHF) facility to produce a time sequence of proton transmission radiographs throughout the dynamic event, a kicker/modulator for extraction from the $50 \mathrm{GeV}$ ring that is capable of generating a string of 25 pulse pairs at arbitrary times within a total time duration of 100 microseconds or more is desired. The full range of desired pulse-train requirements cannot be met with the commonly used pulse-forming cables or networks (PFNs) switched with thyratrons. The preferred modulator design approach at present is a transformer voltage-adder concept with primary-side pulses formed with MOSFET-switched capacitors. This modulator will be a scale-up of an existing modulator that has been developed by Lawrence Livermore National Laboratory for use in DARHT, an electron induction accelerator facility at Los Alamos National Laboratory. Before the voltageadder concept can be adopted for use in AHF, a working prototype that meets the AHF requirements for the pulse voltage, current, rise and fall time, and total pulse number must be built and tested. Additional requirements for pulse-to-pulse flattop height variation and baseline shift must also be met. A development and testing plan for the voltage-adder kicker modulator for AHF is described.
\end{abstract}

\section{INTRODUCTION}

Under the US nuclear weapons Stockpile Stewardship program, The AHF facility has been proposed as a new means of measuring the reliability and safety of nuclear weapons primaries. The goal of the AHF is to follow the hydrodynamic evolution of dense, thick objects that are driven by high explosives by acquiring flash proton radiographic images at multiple times during the event from multiple view angles. The present requirements are for 20 time frames and 12 view angles evenly spaced over $180^{\circ}$. The images in the 12 views must be simultaneous to within a few tens of nanoseconds. The proton pulse format of a proton synchrotron (high instantaneous intensity, multiple short-duration bunches, low average duty cycle) is ideally matched to the requirements of dynamic proton radiography. The energy of the Advanced Hydrotest Facility (AHF) synchrotron is $50 \mathrm{GeV}$, which is the result of a compromise between improved performance and increased cost with increasing energy. Fig. 1 is an AHF facility overview showing an $\mathrm{H}^{-}$linac,

*Research supported by US Dept. of Energy under contracts W-7405-Eng-36 (LANL) and W-7405-Eng-48 (LLNL). showing the booster synchrotron, $50 \mathrm{GeV}$ main ring, transport lines, and magnetic-lens lines radiating from the main firing point. After a beam bunch is extracted from the $50-\mathrm{GeV}$ ring, it is routed either to Firing Point 1 or through a series of splitters into the 12 beamlines that intersect at Firing Point 2. Near-simultaneous illumination in the 12 view angles at Firing Point 2 is ensured by the fact that the beamline lengths are equal except for the small length differences after the final threeway splits.

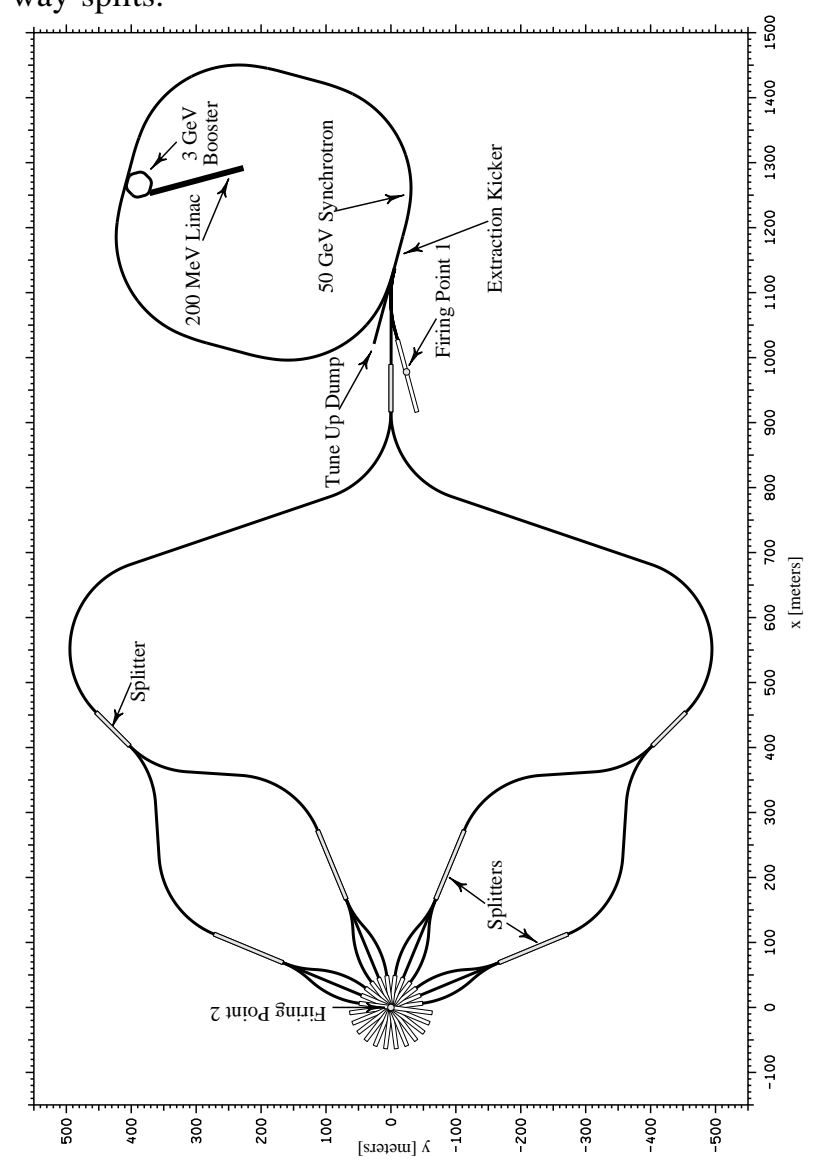

Figure 1: AHF layout.

\section{REQUIREMENTS}

Extraction system requirements are driven by the synchrotron bunch number, desired bunch extraction format, beam rigidity and synchrotron lattice constraints, and beam phase-space considerations. The synchrotron bunch number and extraction format are chosen to meet he requirements of the experimental program. 


\subsection{Bunch Extraction Format}

The center-to center bunch spacing $T_{\text {bunch-bunch }}$ at the time of extraction is $200 \mathrm{~ns}$. The ring revolution time is approximately $5 \mu \mathrm{s}$. In a typical experiment, a series of approximately 10 time frames spaced approximately by integral multiples of ring revolution time would be first taken, followed by a final burst of 10 time frames spaced by $200 \mathrm{~ns}$. The first 10 bunches would be extracted singly, and the last 10 in a single turn. In general, the kicker/modulators must be capable of extracting either individual bunches or groups of adjacent bunches at arbitrary times.

\subsection{Kick Strength and Modulator Voltage Requirements}

In the present extraction scheme, approximately $20 \mathrm{~m}$ of space are available for placement of extraction kickers. With a bump scheme to bring the accelerated beam close to the extraction Lambertson septum magnet, a total kick of $1.8 \mathrm{mrad}$ is required to kick extracted bunches into the deflecting field volume of the septum magnets. This kick can be produced by two separately powered ferrite-free 9$\mathrm{m}$-long parallel-plate transmission-line kickers driven with $\pm 50 \mathrm{kV}$ pulses in a push-pull mode. This kicker type was chosen because it can be designed for a fast rise time and a flat impedance over a wide frequency range. In order to maximize the kick while minimizing the voltage, the kickers are contoured to follow the injected beam envelope. Each side of the kickers presents a 50-ohm matched impedance to the pulse transmission cable when it is powered in this mode, the vertical center plane of the kicker forming a virtual ground. Each side of the kicker is terminated with a 50-ohm impedance to ground. The kickers are driven from the downstream end with respect to the beam direction; when operating in this mode, half of the kick comes from the electric field and half from the magnetic field (setting the beam $\beta=1$, a good approximation at $50 \mathrm{GeV}$ ).

\subsection{Rise- and Fall-Time Requirements}

The rise- and fall-time requirements for the modulator pulses depend on the assumptions made for achievable pulse quality. The length of the modulator pulse flattop needed for extraction of a single beam bunch, defined as the part of the flattop that meets a $\pm 1 \%$ flatness requirement, must be at least twice the length of the kicker divided by the speed of light, plus the beam bunch length. With 9-m kickers and 20-ns beam bunches, the pulse flattop length $\tau_{\text {flat }}$ is $80 \mathrm{~ns}$. The worst-case single bunch extraction case occurs when the extracted bunch has adjacent bunches before and after it that must be left undisturbed in the ring. If the modulator pulse is applied to the downstream end of the kicker just as the previous (unkicked) bunch leaves the kicker, and the fall time of the pulse is completed just before the next bunch enters the kicker, a total of $\tau_{\text {ise }}+\tau_{\text {fall }}=2 T_{\text {bunch-bunch }}-\tau_{\text {flat }}$ can be allowed for the sum of the rise and fall times (this includes any overshoot, ringing etc. in the flattop that exceed the flatness criterion). If adjacent bunches are to be kicked out

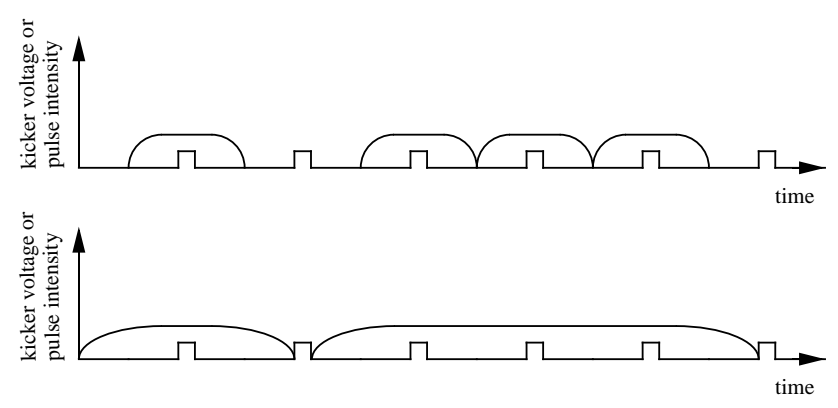

Figure 2: Extraction of adjacent bunches with constantwidth (upper graph) and stretched (lower graph) pulses.

with a single modulator pulse, the flattop must be stretched in length. This is illustrated schematically in Fig. 2. Alternatively, two or more adjacent bunches can be kicked out by applying identical repeated modulator pulses to the kicker. In this case the total modulator pulse width must be shorter in time to allow for fall of one pulse and rise of the next between beam bunches. The allowable sum of rise and fall times then $\tau_{\text {ise }}+\tau_{\text {fall }}=T_{\text {bunch-bunch }}-\tau_{\text {flat }}$. With $T_{\text {bunch-bunch }}=200$ ns and $\tau_{\text {flat }}=80 \mathrm{~ns}, \tau_{\text {ise }}+\tau_{\text {fall }}$ is $120 \mathrm{~ns}$ for the constant-width case and $320 \mathrm{~ns}$ for the stretched-pulse case. Choice between the two operating modes (stretched $v s$. constant-width) will be made after analysis of the effects of measured waveforms from prototype testing, taking into account the fact that the kick given to a proton in the beam bunch is the average of the field strength applied to it as it passes through the kicker.

\subsection{Volt-Second and Current Requirements}

We have taken for the worst case a single $5-\mu$ s stretched pulse plus 15 individual stretched pulses with a flattop of $80 \mathrm{~ns}$ and $50 \mathrm{~ns}$ rise and fall times with an average voltage during the rise time of half of the $50-\mathrm{kV}$ flattop. This gives a total volt-second requirement of $0.35 \mathrm{~V}$-s. Since the load is $50 \mathrm{ohms}$, the flattop current is $1000 \mathrm{~A}$ and the total charge transfer is $7 \times 10^{-3} \mathrm{C}$.

\section{THE VOLTAGE-ADDER CONCEPT}

The present voltage-adder kicker modulator concept (see Fig. 3) is an outgrowth of solid-state pulsed-modulator development for induction accelerators at Livermore. The voltage adder is basically a step-up transformer with many separately powered parallel single-turn primary windings and a single output circuit. The transformer is formed from a stack of annular Metglas magnetic cores. The output circuit is formed by a center cylinder that passes through the center of the core stack and is grounded at the 


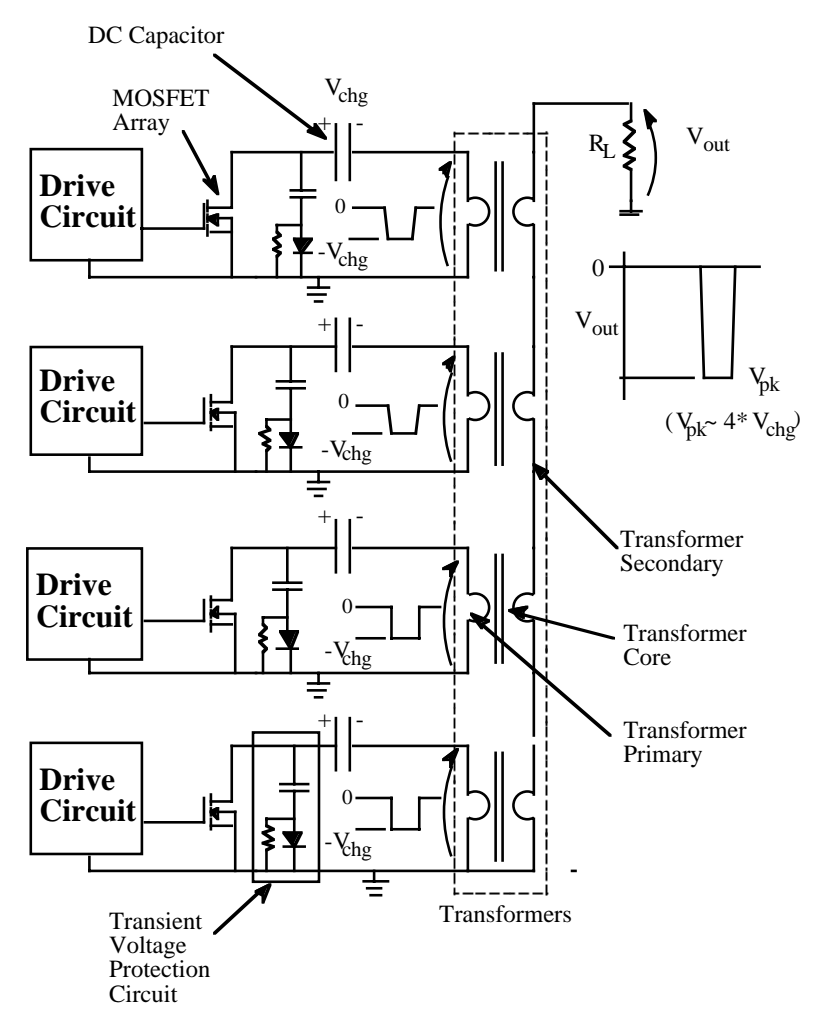

Fig. 3: Simplified circuit schematic for the voltage-adder concept.

bottom of the stack, and by the core housings. Circuit boards with parallel MOSFET switches plug into the spaces between the cores. Each board has 12 parallel MOSFETS and there are two boards per core. MOSFETs with a $1000 \mathrm{~V}$ rating are de-rated to $750 \mathrm{~V}$ for reliability. Energy for the pulses is stored in capacitors on the boards. Intrinsic switching times for the MOSFETS is approximately $15 \mathrm{~ns}$. Production of clean pulses requires near-simultaneous triggering of the MOSFETS with less than $1 \mathrm{~ns}$ jitter in trigger pulse timing. Each core provides approximately $750 \mathrm{~V}$ to the output pulse; for 50 $\mathrm{kV}$ output, capacitors and cores are sized to provide the $0.35 \mathrm{~V}$-s output. Capacitors are allowed to sag approximately $5 \%$ over the pulse train; in order to compensate additional cores and circuits are switched in to compensate for capacitor droop. For this reason approximately 68 cores will be switched initially and 4 additional cores will be switched in succession later in the string of pulses to compensate for capacitor droop. In order to reduce the modulator height two stacks in separate cabinets connected by a solid coaxial line will be used.

\section{DEVELOPMENT PLAN}

A four-stage engineering-development plan for the AHF extraction system kicker/modulator is described below. Stage 1 of the plan is presently under way at LLNL. The plan requires a scale-up from the DARHT voltage of 20 $\mathrm{kV}$ to $50 \mathrm{kV}$ for AHF and the same scale-up in current. The scale-up of volt-seconds is considerably greater: nearly a factor or of 30 . However, rise-time requirements for AHF are less severe: a minimum of $60 \mathrm{~ns}$ for AHF $v s$. $10 \mathrm{~ns}$ for DARHT. Circuit modeling will be performed in parallel with the hardware design and testing in each of the four stages.

Stage 1. Demonstrate full-voltage $(50 \mathrm{kV})$ and fullcurrent (1000A) operation of a single modulator based on DARHT-II voltage-adder magnetic cores with a 50-ohm dummy load. The goal of Stage 1 is to demonstrate that the modulator can meet the AHF pulse rise-time and ripple requirements for one or two pulses. Demonstration of the production of the full set of AHF pulses will not be performed in Stage 1 since the voltage-second capability DARHT-II cores and stored energy of the on-board board capacitors do not meet AHF requirements. Some voltage sag in the pulse flattop is therefore expected. However, since it is based as much as possible on existing hardware, the Stage 1 test will address the key issues of pulse rise time and ripple at the earliest reasonable time. Some lifetime and component failure-rate testing will also be done in this stage.

Stage 2. Perform modulator tests with a dummy lowresistance load (sized to give 1000 A with the nominal $800 \mathrm{~V}$ energy-storage capacitor charge voltage), using a set of circuit boards and a single core set. The core will be sized to meet the full volt-second capability (per core) required for AHF operation. The circuit boards will be further modified to include increased on-board energystorage capacitance. The goal of this stage to verify predictions of core magnetic behavior, including magnetization current, and to produce a complete string of AHF-format pulses with the required rise time and flattop duration, sag, and ripple

Stage 3. Using the cores and circuit boards developed in Stage 2, build a single modulator that meets all of the AHF requirements (voltage, current, pulse rise time, flattop ripple and flatness, full AHF pulse format, etc.) and test it with a length of transmission cable and a dummy 50-ohm load. The goal of Stage 3 is to demonstrate a working full-scale modulator that meets AHF requirements.

Stage 4. Build a second modulator that is the same as that of Stage 3, but of opposite voltage polarity, and connect the pair in a push-pull configuration with 50 -ohm transmission lines to a prototype double-sided transmission-line-type kicker with 50-ohm terminations on each side. The goal of Stage 4 is to demonstrate that the full kicker/modulator system meets all AHF requirements. Tests will be performed without a proton beam but with voltage and current measurements at the kicker to verify that the system meets AHF requirements for voltage, current, pulse rise time, flattop ripple and flatness), including reflections at the kicker, etc. 